\begin{tabular}{ll} 
POS & PROCEEDINGS \\
\hline
\end{tabular}

\title{
New Developments in FormCalc 8.4
}

\section{Christoph Groß}

Institut für Theoretische Physik und Astrophysik, Universität Würzburg, D-97074 Würzburg, Germany

E-mail: chrisgr90@gmx.net

\section{T. Hahn*}

MPI für Physik, Föhringer Ring 6, D-80805 Munich, Germany

E-mail: hahn@mpp.mpg.de

\section{Sven Heinemeyer}

Instituto de Física de Cantabria (CSIC-UC), E-39005 Santander, Spain

E-mail: Sven.Heinemeyer@cern.ch

\section{Federico von der Pahlen}

Instituto de Física, Universidad de Antioquia, Calle 70 No. 52-21, Medellín, Colombia

E-mail: fp@gfif.udea.edu.co

\section{Heidi Rzehak}

Albert-Ludwigs-Universität Freiburg, Physikalisches Institut, D-79104 Freiburg, Germany

E-mail: Heidi.Rzehak@cern.ch

\section{Christian Schappacher}

Institut für Theoretische Physik, Karlsruhe Institute of Technology, D-76128 Karlsruhe,

Germany (former address)

E-mail: schappacher@kabelbw.de

We present new developments in FeynArts 3.9 and FormCalc 8.4, in particular the MSSMCT model file including the complete one-loop renormalization, vectorization/parallelization issues, and the interface to the Ninja library for tensor reduction.

Loops and Legs in Quantum Field Theory - LL 2014,

27 April - 2 May 2014

Weimar, Germany

\footnotetext{
*Speaker.
} 


\section{Introduction}

FeynArts [1] and FormCalc [2] are Mathematica packages for the generation and simplification of Feynman diagrams. In a first step, FormCalc reads diagrams up to one-loop order generated by FeynArts and returns a simplified symbolic result which can be inspected or modified using regular Mathematica commands as well as several FormCalc functions and options for analytic manipulations, such as the application of Fierz identities. For efficient numerical evaluation, e.g. of the cross-section, the analytical expressions can then be written out as highly optimized subroutines in Fortran or C, complemented by a library of utility functions. The generated code is largely selfcontained. It uses simple Fortran programs to set the inputs rather than (static) parameter cards, which would be limited to a certain class of models. The numeric calculation can also be interfaced back to Mathematica through MathLink, such that e.g. the cross-section becomes available as a Mathematica function of its input parameters.

This note presents new features and improvements in FeynArts 3.9 and FormCalc 8.4:

- The MSSMCT model file including the complete one-loop renormalization of the complex MSSM.

- Run-time selection of renormalization schemes.

- Vectorization/parallelization issues in FormCalc, LoopTools, and Cuba.

- Ninja interface for FormCalc.

\section{The MSSMCT Model File}

The full renormalization prescriptions of the MSSMCT model file [3] cannot be reproduced in this limited space. Rather, we focus on a few conceptually important issues which also required changes in FeynArts and FormCalc.

\subsection{The Higgs-boson Sector}

Higher-order corrections are phenomenologically very important in the Higgs sector. Indiscriminately including them on propagators and vertices risks upsetting the relations necessary for the proper cancellation of UV and IR divergences, however, since this mixes orders in perturbation theory. The masses on the Higgs propagtors should ideally be consistent with the Higgs mixing angle $\alpha$ parameterizing the vertices, but this conflicts with the fact that mixing can occur between all three states $h, H, A$ at loop level, which is not expressible through a single angle $\alpha$.

We opted therefore to formulate the vertices with the tree-level $\alpha$ and insert tree-level masses on loop propagators but use loop-corrected masses on all other propagators. At the level of the Feynman rules it is not possible to generally avoid incomplete cancellations due to a mismatch between tree-level and loop-corrected masses, though there are typically process-specific solutions. Our recommendation is to test UV and IR finiteness with loop-corrected masses and revert to treelevel masses as far as necessary.

On the technical side, FeynArts needed to be extended to allow a detailed choice of the masses inserted on different types of propagators. For example, the declaration of the light CP-even Higgs boson $h$ includes the two mass specifiers, 


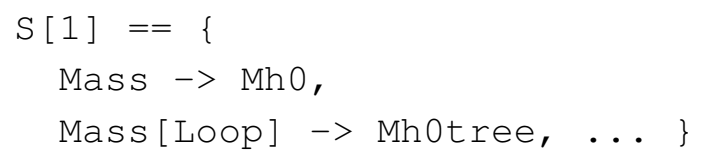

\subsection{The Fermion/Sfermion Sector}

The model file is presently limited to minimal flavor violation in the sfermion sector, which means that for non-trivial CKM matrix there is a slight imbalance between fermions and sfermions; for example, the $b$-quark has an admixture from $d$ and $s$ while the $\tilde{b}$ does not. Because this violates delicate supersymmetric relations, reactions involving squarks (in particular external ones) may not become finite and we have therefore chosen to turn CKM mixing off by default. It can be enabled by setting $\$ C K M=$ True.

\subsection{Run-time Renormalization Scheme Selection}

In the sfermion and chargino/neutralino sectors the available degrees of freedom do not allow all physical quantities to be chosen on-shell simultaneously. Solving for the dependent parameters typically yields results which become numerically unstable in certain regions of parameter space, e.g. because of some combination of mixing-matrix elements in the denominator. For example, in the CCN schemes (two charginos and one neutralino on-shell) the renormalization constants $\delta M_{2}$ and $\delta \mu$ diverge in regions where $|\mu| \approx\left|M_{2}\right|$ (gaugino-Higgsino mixing close to maximal).

For this reason one would like to switch between different schemes at run-time, say while scanning over parameter space. Since the dependent parameters vary from scheme to scheme, straightforward if-statements such as dMUE1 $=$ IndexIf $\left[\right.$ cond $\left., \delta \mu^{\mathrm{A}}, \delta \mu^{\mathrm{B}}\right]$ may contain overlapping dependencies, however, and cannot naively be ordered for evaluation in Fortran. For example,

dMUE $1=$ IndexIf $\left[\right.$ cond $\left., \delta \mu^{A}\left(\delta M_{1}^{A}\right), \delta \mu^{B}\right] ;$

dMino11 = IndexIf [cond $\left., \delta M_{1}^{A}, \delta M_{1}^{B}\left(\delta \mu^{B}\right)\right]$;

cannot be ordered as it stands, for $\delta \mu$ is a function of $\delta M_{1}$ in scheme $A$, but oppositely in $B$. The solution was to have FormCalc join the 'if' and 'else' parts of all if-statements with identical conditions, upon which the statements in each branch can be ordered correctly:

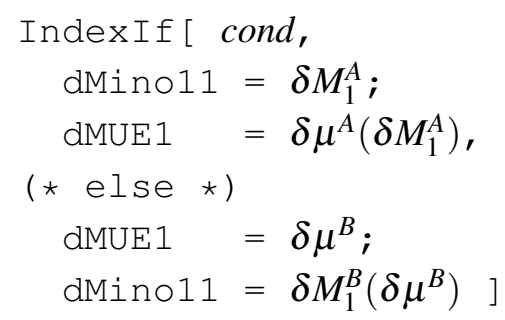

On the user side, scheme switching is available in MSSMCT as e.g.

\$InoScheme $=$ IndexIf $[$ cond, $\operatorname{CNN}[2,1,3], \operatorname{CCN}[1]]$

where cond might be Abs [Abs [MUE]-Abs [Mino2] $]<50$.

Note that a renormalization-scheme switch in principle requires a corresponding transition of the affected parameters from one scheme to the other (not yet included by default) for a fully consistent interpretation of the results. 
In the light of recent activities to automate the generation of Feynman rules also for oneloop counter terms [4], it should clearly be stated that the MSSMCT model file goes far beyond simple $\overline{\mathrm{DR}}$ renormalization for the BSM part. As outlined above, SUSY relations and restrictions due to the available degrees of freedom are taken into account, and renormalization conditions can be chosen to obtain physically meaningful renormalized parameters over the entire parameter space. We believe this much wider program requires physical understanding and cannot easily be automated.

\section{Vectorization/Parallelization Issues}

\subsection{Vectorization of the Helicity Loop}

The assembly of the squared matrix element in FormCalc can be sketched as in the following figure, where the helicity loop sits at the center of the calculation:

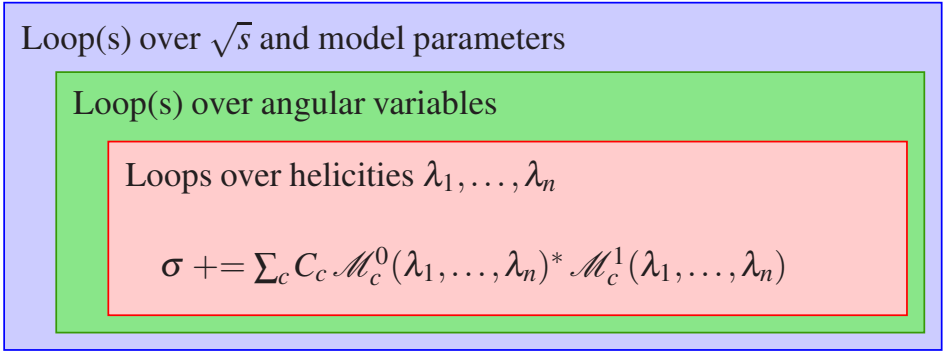

The helicity loop is not only strategically the most desirable but also the most obvious candidate for concurrent execution, as FormCalc does not insert explicit helicity states during the algebraic simplification [5]. Such a design is known as Single Instruction Multiple Data (SIMD) in computer science and is conceptually easy to vectorize. With an AVX-capable i7, a speedup of 3.7 out of a theoretical 4 for the helicity loop could be achieved.

Vectorization is available in both Fortran (using Fortran 90's vector syntax) and C99 (through macros emitting explicit SSE3/AVX instructions) and is enabled by default according to the capabilities of the machine on which configure is run. To configure the code for an arbitrary machine (i.e. without vectorization), the --generic flag must be used with configure. More in detail, the automatically determined maximum hardware vector length is written to simd.h and the user can control in distrib.h whether to include simd.h (default) or define a different vector length in the SIMD preprocessor variable (zero for no vectorization).

Since the OPP libraries are currently not capable of handling vector-valued numerator functions, SIMD must be set to zero in distrib.h when using the CutTools [6], Samurai [7], or Ninja [8] libraries.

\subsection{Dropping Negligible Helicity Combinations}

The helicity sum can further be improved by observing that typically many terms contribute to the final result only negligibly. While helicity combinations that give an exact zero result could in principle be identified analytically, this is not really straightforward due to the way the amplitudes are composed of abbreviations, and it is far less straightforward for small but non-zero (e.g. masssuppressed) combinations. 
The FormCalc-generated code adopts a numerical strategy instead: It observes the terms in the helicity sum for a (smallish) number of phase-space points, then identifies and suppresses computation of the negligible ones for all further phase-space points.

Two environment variables control the procedure: FCHSELN specifies the number $n$ of phasespace points to be observed before taking the decision and FCHSELEPS specifies how much smaller the contribution of an individual helicity contribution must be relative to the maximum to be neglected, i.e. a helicity combination $\{\lambda\}$ is dropped if

$$
X_{\{\lambda\}}<\varepsilon \max _{\{\lambda\}} X_{\{\lambda\}} \quad \text { where } \quad X_{\{\lambda\}}=\sum_{i=1}^{n}\left|\mathscr{M}_{\{\lambda\}}^{(i)}\right| .
$$

\subsection{Concurrency Issues in Loop Tools}

LoopTools [2] uses a cache to speed up calculation. Originally this was done for tensor coefficients only, for which there is both a significant overlap of intermediate results in the computation and a high chance that once one coefficient is called, related ones will be needed as well. Recently the cache system was extended to scalar integrals, too, so that $\mathrm{CO}(\ldots)$ (formerly not cached) and $\mathrm{COi}(\mathrm{ccO}, \ldots)$ (formerly cached) are now identical. Depending on the application, the cache can easily make an order-of-magnitude difference in run time.

On the other hand, LoopTools was not thread-safe because of the cache. (Indeed, the actual computation of the scalar integrals in Fortran with its use of common blocks etc. was not really thread-safe either.) In a concurrent environment this is most dangerous, as it may silently lead to wrong results.

Thread-safety has been achieved by serializing cache writes through mutexes. It was also for this reason, to make them thread-safe, that the scalar functions were moved into the cache system. The uncached versions (not thread-safe) are still available as Aputnocache, Bputnocache, COnocache, DOnocache, EOnocache.

With serialization, cache management has become a strategically even more important task. Although LoopTools performs efficient binary-tree cache lookups, indiscriminate build-up of the cache does not only cost memory but performance as well. Care should be taken therefore to flush the cache whenever a lookup will not reasonably be successful anymore, e.g. when moving to a different point in phase or parameter space, using the clearcache, markcache, and restorecache calls.

\subsection{Vectorization and GPU Issues in Cuba}

Cuba [9] is a library for multidimensional numerical integration. From version 3 on it automatically parallelizes sampling of the integrand using fork/wait. The Cuba calls were extended in version 3.3 to include a new argument, nvec, through which one can have the integrator deliver more than one point per call to the integrand and e.g. vectorize the computation this way.

Another interesting option is to do massively parallel sampling on a GPU. Re-writing the integrand function for execution on the GPU is necessarily a task of the user (i.e. cannot be automatically done by Cuba). GPUs have a somewhat different thread model than the CPU, however, and so the strategy of determining the slices to be sampled by each core in Cuba 3.x was barely sufficient (at least not very convenient) for use with a GPU. Cuba 4.0 redresses this by distinguishing 
'accelerators' (GPU cores) and 'cores' (CPU cores, same as in 3.x) and distributes samples differently for either kind; most importantly it divides a core's share of points into batches no larger than the GPU can handle.

Smaller batches are useful even for CPU cores, for they effectively load-level the sampling. To sample 50000 points on four cores, for example, Cuba 3.x would distribute 12500 points to each core. This is optimal only if the evaluation of the integrand is equally fast at all points, however, otherwise it is smarter to distribute smaller batches of a user-defined size, say 2000, which reduces the chance of a bottleneck at a very reasonable increase in communication overhead.

\section{Ninja Interface}

Ninja [8] is a library for tensor reduction based on unitarity methods. Unlike the 'traditional' OPP libraries CutTools [6] and Samurai [7], Ninja does not rely on sampling the numerator alone but performs a Laurent expansion of the integral and is thereby able to reconstruct the numerator tensor using far fewer samples and in a numerically more stable way. To pull off this feat, Ninja requires several expansions of the numerator function $N\left(q^{\mu}, \mu^{2}\right)$ (with loop momentum $q^{\mu}$ and renormalization scale $\mu$ ) in the variables $t, x$, and $\mu^{2}$ as follows:

- the numerator itself: $N\left(q^{\mu}, \mu^{2}\right)$,

- the $\mu$ expansion, for computation of the box coefficients: $N\left(t v_{\perp}^{\mu}, t^{2} v_{\perp}^{2}\right)$,

- the T3 expansion, for computation of the triangle and tadpole coefficients:

$$
N\left(v_{0}^{\mu}+t v_{3}^{\mu}+\frac{\beta+\mu^{2}}{t} v_{4}^{\mu}, \mu^{2}\right)
$$

- the T2 expansion, for computation of the bubble coefficients:

$$
N\left(v_{1}^{\mu}+x v_{2}^{\mu}+t v_{3}^{\mu}+\frac{\beta_{0}+\beta_{1} x+\beta_{2} x^{2}+\mu^{2}}{t} v_{4}^{\mu}, \mu^{2}\right) \text {. }
$$

FormCalc generates each of these numerator expansions as an independent subroutine which computes the coefficients of $t^{j} x^{k} \mu^{2 l}$ in the ranges of $\{j, k, l\}$ needed by Ninja as a function of the remaining parameters $v_{i}, \beta_{i}$.

\section{Summary}

FeynArts 3.9 (http : //feynarts.de) and FormCalc 8.4 (http : //feynarts.de/formcalc) have many new and improved features, most notably the MSSMCT model file including the full one-loop renormalization of the MSSM, support for vectorization/parallelization, and the interface to the Ninja library.

\section{References}

[1] Hahn T, 2001, Comput. Phys. Commun. 140418 [hep-ph/0012260].

[2] Hahn T, Pérez-Victoria M, 1999, Comput. Phys. Commun. 118153 [hep-ph/9807565]. 
[3] Fritzsche T, Hahn T, Heinemeyer S, von der Pahlen F, Rzehak H, Schappacher C, 2014, Comput. Phys. Commun. 1851529 [arXiv:1309.1692]. Heinemeyer S, et al., these proceedings.

[4] Degrande C, 2014, arXiv:1406.3030.

[5] Hahn T, 2003, Nucl. Phys. Proc. Suppl. 116363 [hep-ph/0210220].

[6] Ossola G, Papadopoulos C, Pittau R, 2008, JHEP 0803042 [arXiv:0711.3596].

[7] Mastrolia P, Ossola G, Reiter T, Tramontano F, 2010, JHEP 1008080 [arXiv:1006.0710].

[8] Peraro T, 2014, arXiv:1403.1229.

[9] Hahn T, 2005, Comput. Phys. Commun. 16878 [hep-ph/0404043]. 